Article

\title{
Crambescidin-816 Acts as a Fungicidal with More Potency than Crambescidin-800 and -830, Inducing Cell Cycle Arrest, Increased Cell Size and Apoptosis in Saccharomyces cerevisiae
}

\author{
Juan A. Rubiolo ${ }^{1,2}$, Eva Ternon ${ }^{3}$, Henar López-Alonso ${ }^{1}$, Olivier P. Thomas ${ }^{3}$, Félix V. Vega ${ }^{2}$, \\ Mercedes R. Vieytes ${ }^{2}$ and Luis M. Botana ${ }^{1, *}$
}

1 Department of Pharmacology, Faculty of Veterinary, University of Santiago de Compostela (USC), Campus Lugo, Lugo 27002, Spain; E-Mails: ja.rubiolo@usc.es (J.A.R.); henar.lopez@usc.es (H.L.-A.)

2 Department of Physiology, Faculty of Veterinary, University of Santiago de Compostela (USC), Campus Lugo, Lugo 27002, Spain; E-Mails: felixvictor.vega@usc.es (F.V.V.); mmercedes.rodriguez@usc.es (M.R.V.)

3 Nice Institute of Chemistry-PCRE, UMR 7272 CNRS, Faculty of Sciences, University of Nice Sophia Antipolis, Parc Valrose 06108 Nice, France; E-Mails: eva.ternon@unice.fr (E.T.); olivier.thomas@unice.fr (O.P.T.)

* Author to whom correspondence should be addressed; E-Mail: luis.botana@usc.es; Tel.: +34-9822-52195; Fax: +34-98282-2233.

Received: 29 August 2013; in revised form: 25 October 2013 / Accepted: 29 October 2013 / Published: 8 November 2013

\begin{abstract}
In this paper, we show the effect of crambescidin-816, -800 , and -830 on Saccharomyces cerevisiae viability. We determined that, of the three molecules tested, crambescidin- 816 was the most potent. Based on this result, we continued by determining the effect of crambescidin-816 on the cell cycle of this yeast. The compound induced cell cycle arrest in G2/M followed by an increase in cell DNA content and size. When the type of cell death was analyzed, we observed that crambescidin-816 induced apoptosis. The antifungal effect indicates that crambescidins, and mostly crambescidin-816, could serve as a lead compound to fight fungal infections.
\end{abstract}

Keywords: crambescidine-816; antifungal; cell cycle arrest; apoptosis 


\section{Introduction}

Marine sponges are among the most important producers of secondary metabolites with antimicrobial effect. After centuries of evolutionary pressure, these sessile marine filter feeders have developed a very efficient chemical arsenal against virus, bacteria, and eukaryotic organisms. More than 200 new metabolites from this group of animals are reported each year and more than 5300 different secondary metabolites are known from sponges [1,2]. Meanwhile, in the last decade, many compounds with antifungal activity have been isolated from sponges (for comprehensive reviews see [3-6]) indicating that this group of organisms is a valuable source of this type of compounds. The sponge Crambe crambe commonly found in the rocky costs of the Mediterranean sea, is capable of inducing necrosis of other sponge tissues when they are kept in contact [7], and was initially reported to produce potent antibacterial and antifungal compounds [8]. Additionally, metabolites of this highly toxic sponge have proven to have antifouling activity against microfoulers and invertebrate larvae [9]. Two families of guanidine alkaloids have been isolated from Crambe crambe, namely crambescidins and crambescins. Some of these have been shown to have mid nanomolar cytotoxicities against human tumor derived cell lines [10]. Crambescidin-800 (C800) induces differentiation of K562 leukemia cells leading to cell cycle arrest in S-phase [11]. C800 was initially identified as a promising antiviral and antifungal compound [10], and later purified from Monanchora unguifera in the search of antifungal leads, after a high-throughput screening with the aim of discovering biologically active small molecules, using Saccharomyces cerevisiae as model organism. This compound presented an $\mathrm{IC}_{20}$ in liquid culture of $0.83 \mu \mathrm{M}$, similar to that of the most active selected compounds from the NCI diversity set (collection of 2000 synthetic small molecules selected from the full NCI screening collection) [12]. Another crambescidin isolated from Crambe crambe is crambescidin-816 (C816). This compound has been shown to exert a $\mathrm{Ca}^{2+}$ antagonistic activity with higher potency than nifedipine $[13,14]$. It is also active against human colon carcinoma cells [13].

Due to the problems associated with current antifungal drugs, and the increasing number of invasive fungal infections in immunocompromised patients, a new generation of antifungal drugs is urgently needed. Since crambescidin- 800 has shown promising antifungal activity, and based on the data available for crambescidin- 816 showing that it has a higher activity than crambescidin- 800 in eukaryotic cells, we tested the antifungal effect of crambescidin-816 in comparison with crambescidin- 800 and another of its family members -830 (C830, Figure 1$)$ in $S$. cerevisiae, which is recognized to be a good model for antifungal drug development [15]. We herein show that crambescidin-816 reduces cell viability in $S$. cerevisiae inducing an increment in cell size and DNA content, and apoptosis. The potency of this compound is 10 times higher than that of crambescidin- 800 and 4 times higher than that of crambescidin- 830 . 
Figure 1. Chemical structures of crambescidin-816, -800 , and -830 .

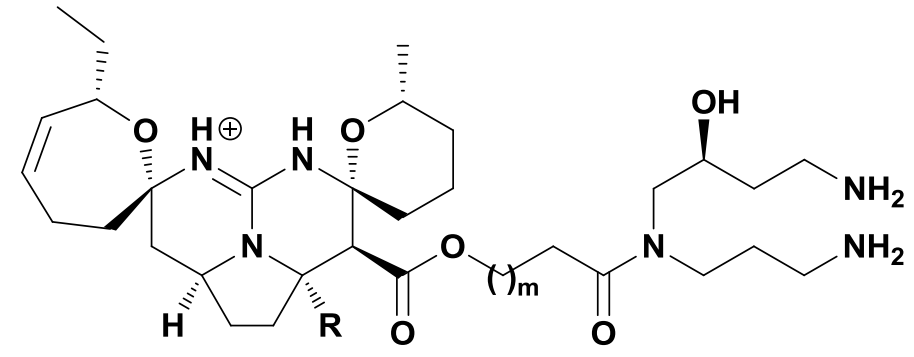

$\begin{array}{lll} & \text { m } & \text { R } \\ \text { Crambescidin-800: } & 14 & \text { H } \\ \text { Crambescidin-816: } & 14 & \mathrm{OH} \\ \text { Crambescidin-830: } & 15 & \mathrm{OH}\end{array}$

\section{Results and Discussion}

\subsection{C816 Is More Potent on S. cerevisiae than C800 and C830, and Has a Fungicidal Effect}

We compared the effect of C816, C800 and C830 on S. cerevisiae cell growth in order to determine their relative potencies. We observed that the most potent molecule was C816 followed by C830 and C800 with $\mathrm{MIC}_{50}$ (the antimicrobial concentration the inhibits $50 \%$ of the growth) at $24 \mathrm{~h}$ of $0.557(95 \%$ confidence interval $=0.5116$ to 0.608$), 1.650(95 \%$ confidence interval $=1.374$ to 2.011$)$, and $4.316(95 \%$ confidence interval $=4.210$ to 4.427$) \mu \mathrm{M}$ respectively. C816 was almost 10 times more potent than C800 (Figure 2).

Figure 2. Determination of the $\mathrm{MIC}_{50}$ for C800, C816 and C830 in S. cerevisiae after $24 \mathrm{~h}$. The optical density determined for each treatment was plotted against the concentrations tested, expressed in molarity. The mean $(n=4) \pm \mathrm{SD}$ for each treatment is shown.

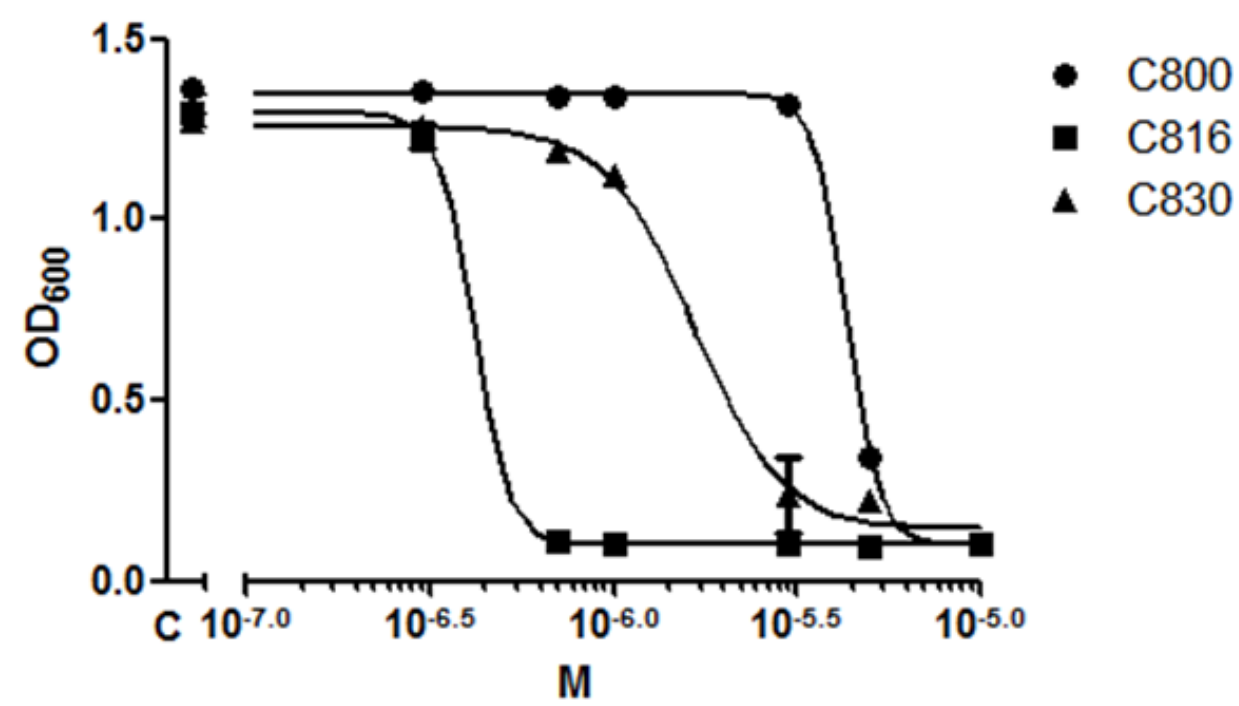

As previously stated in the introduction, C800 has been previously identified as a potent antifungal agent with an $\mathrm{IC}_{20}$ of $0.83 \mu \mathrm{M}$ [12]. At the concentration reported by these authors we observed no 
effect on $S$. cerevisiae cell growth after $24 \mathrm{~h}$. This could be due to the different approaches used for the determination of the effect of the tested compound on cell growth. As shown below, C816 induces a delay of cell growth after short time periods, which is not observed after $24 \mathrm{~h}$. This could explain why the cited authors observed an inhibition of $20 \%$ of cell growth at a concentration of $0.83 \mu \mathrm{M}$ while we determined a minimum inhibitory concentration of $4.316 \mu \mathrm{M}$ after $24 \mathrm{~h}$. An increased lag phase, induced by $\mathrm{C} 800$ could produce an $\mathrm{IC}_{20}$ (defined as the concentration in which the doubling time of the DMSO control divided by the doubling time with compound was equal to 0.8) lower than that observed after incubation for longer time periods. Also, a difference resulting as a consequence of using different strains of $S$. cerevisiae cannot be ruled out. We continued our study with the most potent compound, C816.

To investigate the effect of $\mathrm{C} 816$ on $S$. cerevisiae growth, cultures of this organism were exposed to C816 up to $24 \mathrm{~h}$, and cell growth was monitored measuring the OD of the cultures. We determined that the tested compound inhibited S. cerevisiae growth in a dose dependent manner (Figure 3). At the two highest concentrations tested no cell growth was observed up to $24 \mathrm{~h}$. Lower concentrations (100 and $300 \mathrm{nM}$ ) were capable to delay the growth of the culture after the first $15 \mathrm{~h}$ but finally the cultures reached the same density as control cells after $24 \mathrm{~h}$.

Figure 3. Growth inhibition of $S$. cerevisiae cultures treated with various doses of C816 (100-1000 nM). No growth was observed in cultures treated with 700 and $1000 \mathrm{nM} \mathrm{C816.}$

The mean $(n=4) \pm \mathrm{SD}$ for each treatment is shown.

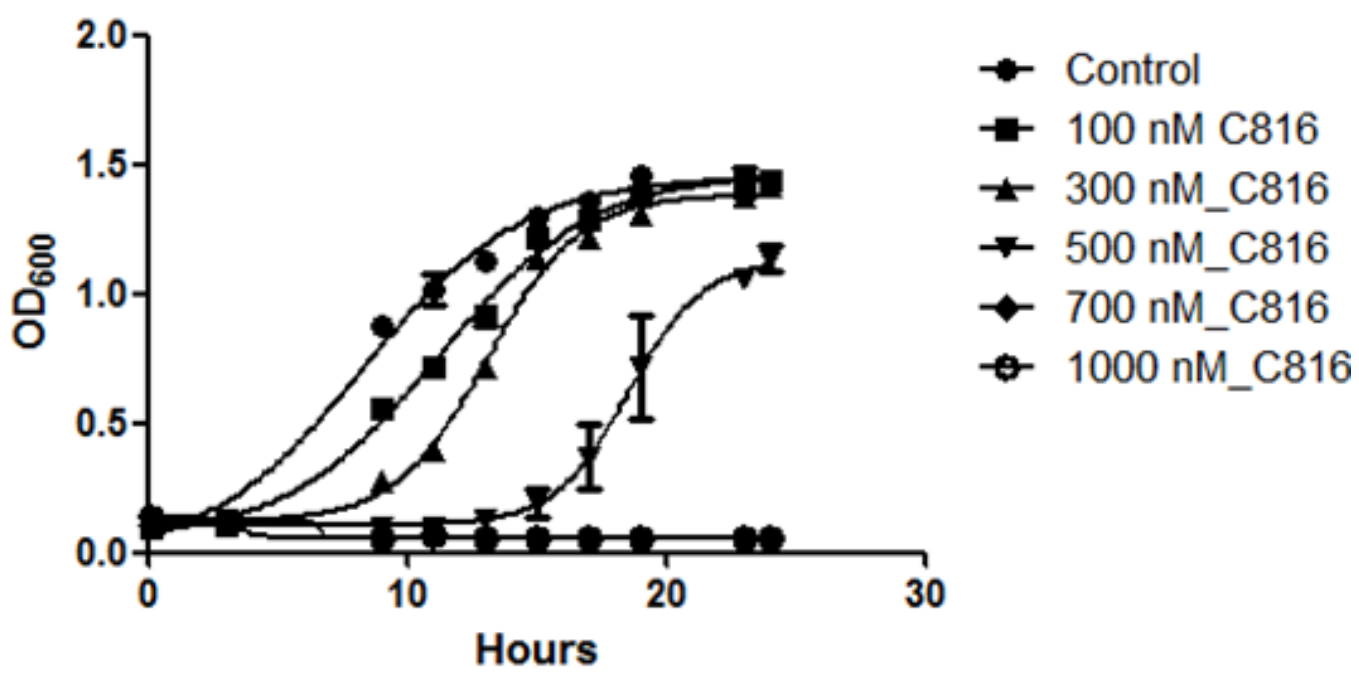

We then assayed if C816 was a fungistatic or if it acted as a fungicide in $S$. cerevisiae. Cells incubated with 300,700 and $1000 \mathrm{nM} \mathrm{C} 816$ for 3 or $6 \mathrm{~h}$ and then cultured in toxin free medium up to $24 \mathrm{~h}$ (see Experimental Section for a detailed explanation of culture conditions) presented a reduced viability at both times tested (Figure 4). Cells exposed to C816 for 6 h showed a reduced growth when compared to those treated with the compound for $3 \mathrm{~h}$. In both cases, a dose dependent response was observed.

The results show that C816 is cytotoxic for $S$. cerevisiae. After $24 \mathrm{~h}$ the cultures treated with the highest concentration tested, reached approximately half of the growth seen in controls. These results indicate that $\mathrm{C} 816$ acts as a fungicidal and not as a fungistatic. 
Figure 4. Determination of the fungicidal effects of C816 on $S$ cerevisiae. Cultures were treated with 500,700 and $1000 \mathrm{nM} \mathrm{C} 816$ for 3 or $6 \mathrm{~h}$. After toxin treatment cells were washed and kept in culture for another $24 \mathrm{~h}$. Culture growth was determined measuring the $\mathrm{OD}$ at $600 \mathrm{~nm}$. Significant differences respect to control $* p<0.01, n=4$.

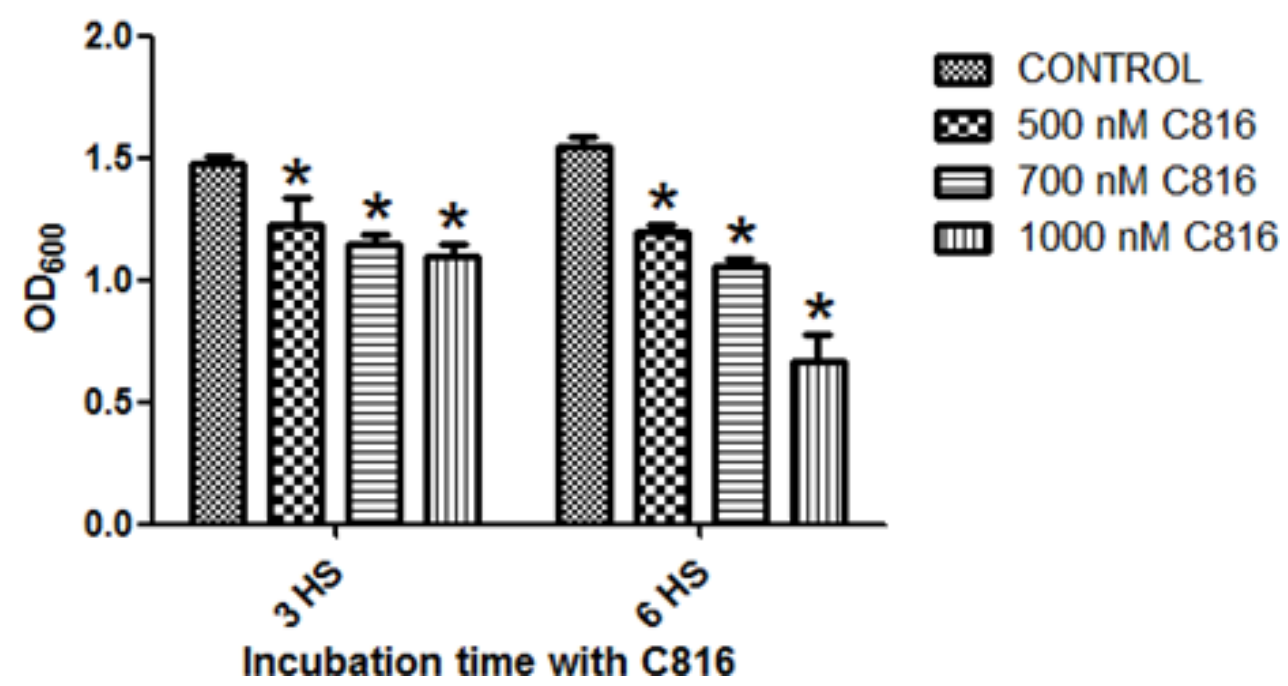

\subsection{C816 Induces Cell Cycle Arrest and an Increase in S. cerevisiae DNA Content}

To assay if C816 affected the cell cycle of $S$. cerevisiae, cultures of these cells were treated with C816 and then studied by imaging flow cytometry. Examples of histograms obtained are shown in Figure 5a. We observed what initially appeared as cell cycle arrest in G2/M in the first $2 \mathrm{~h}$ of culture as determined by the increased population of cells in this phase of the cell cycle for cultures treated with 700 and $1000 \mathrm{nM} \mathrm{C816.} \mathrm{Longer} \mathrm{incubation} \mathrm{periods} \mathrm{indicated} \mathrm{that} \mathrm{C816} \mathrm{induced} \mathrm{the} \mathrm{appearance}$ of cells with a broad range of DNA content. We observed a population of cells with ploidy higher than $4 \mathrm{C}$, which was higher after $6 \mathrm{~h}$ of incubation with 700 and $1000 \mathrm{nM} \mathrm{C} 816$ and after $10 \mathrm{~h}$ of incubation with $300 \mathrm{nM} \mathrm{C816}$ (Figure 5a). No difference was observed after $24 \mathrm{~h}$. At this time point, an increase in cells with a DNA content below of $1 \mathrm{C}$ was detected, which is indicative of apoptosis (Figure 5b). Cells with up to $32 \mathrm{C}$ were detected at the two highest concentrations tested.

The increased DNA content in C816 treated cells could be due to increased mitochondrial DNA content [16]. A similar effect has been observed in $S$. cerevisiae after treatment with the microtubule-depolymerizing drug nocodazole, a compound that induced mitotic cell death with apoptotic-like features [17]. On the other hand, cell death could be a consequence of an abnormal morphogenetic response in the presence of C816 making the cells commit new rounds of DNA replication in the absence of cytokinesis, or cell cycle arrest in anaphase. It has been previously shown that S. cerevisiae can replicate their DNA in the absence of cytokinesis [18], as consequence of septa mislocalization $[19,20]$. This raises the possibility that crambescidins could interfere with cell wall synthesis or organization. The cortical actin cytoskeleton is essential for the development of a functional cell wall and is localized at particular areas at different stages of the cell cycle [21,22]. We have observed that C816 interferes with tumor cell actin cytoskeleton [23], so an effect of this compound on yeast cytoskeleton is also a possibility. Further research is needed to confirm these assumptions. 


\subsection{C816 Induces an Increase in Cell Size}

The images obtained after imaging flow cytometry showed, besides an increase in DNA content, an increment in cell size that appeared early after treatment (Figure 6a). This was also observed after inspection of the cultures by bright field microscopy (Figure 6b).

An increase of cell size is expected since the higher DNA content requires an augmented cell size to accommodate the genetic material.

Figure 5. C816 induces an increase in the DNA content in S. cerevisiae. (a) Histograms obtained after flow cytometry of $S$. cerevisiae treated with C816 for 2, 4, 6, 10 and $24 \mathrm{~h}$. Marked with a rectangle are the cells with 1C, 2C cell cycle distribution. (b) Analysis of cell population distributions after the different treatments performed. Each treatment was assayed in triplicate, and two experiments were performed. The mean $(n=3) \pm \mathrm{SD}$ for each treatment is shown.
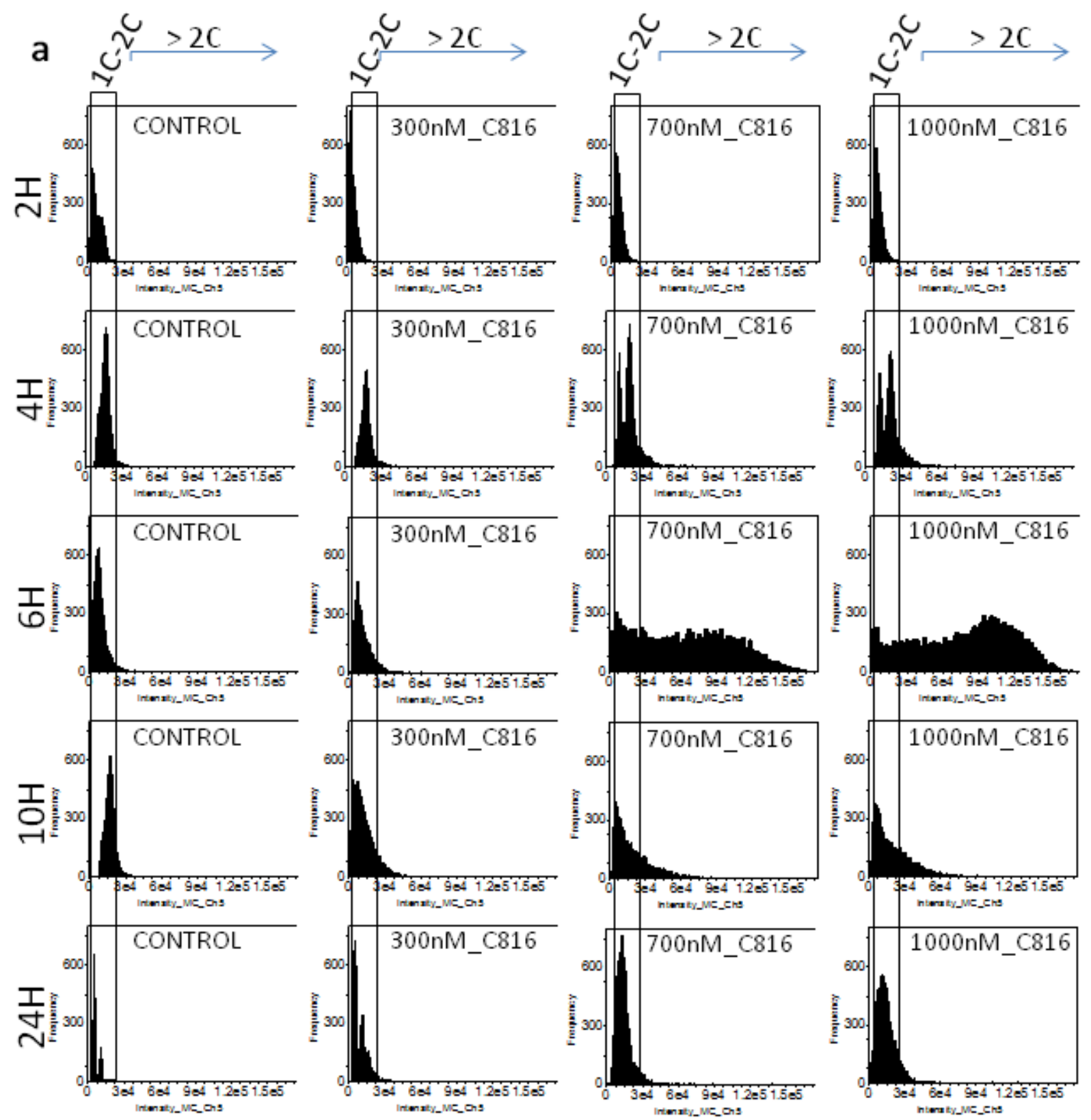
Figure 5. Cont.

b

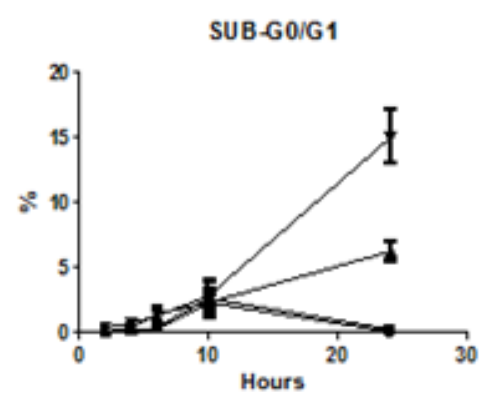

2C

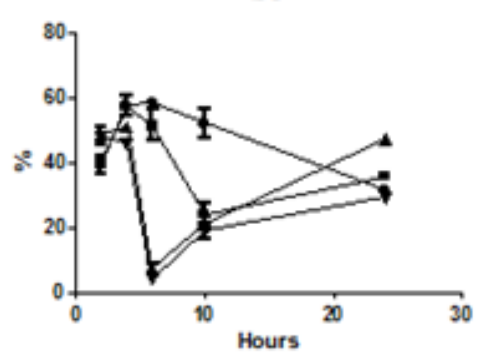

$1 C$

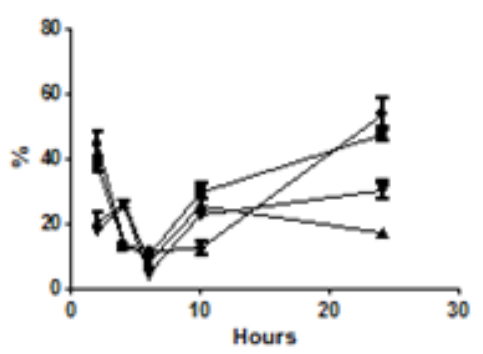

$>2 \mathrm{C}$

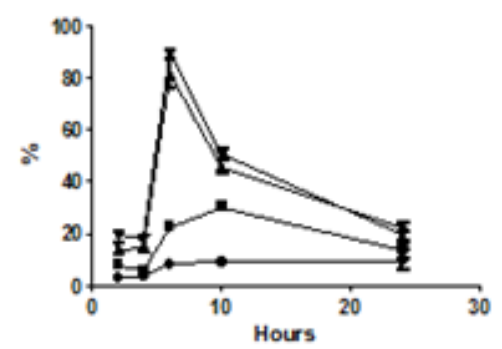

s

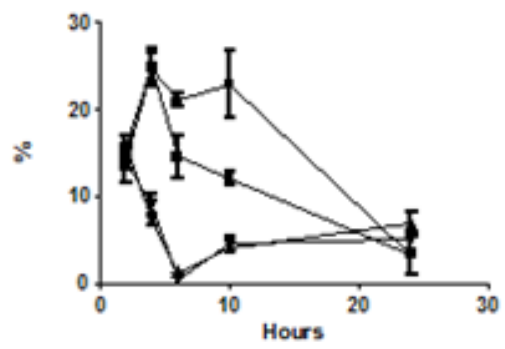

- CONTROL

- 300 nM_C816

× 700 nM_C816

- 1000 nM_C816

Figure 6. C816 induces an increment of the cell size in S. cerevisiae. (a) Representative images of cells in the different populations of the cell cycle after treatment with C816 obtained by imaging flow cytometry. Control samples showed cell with 1 and 2C, while C816 treatment induced the appearance of cells with 4, 8, 16 and 32C. (b) Bright field microscopy of control and C816 treated $S$. cerevisiae. An increase in cell size can be observed in treated cells, especially in cells treated with 700 and $1000 \mathrm{nM} \mathrm{C816.}$ Scale bar $=10 \mu \mathrm{m}$.
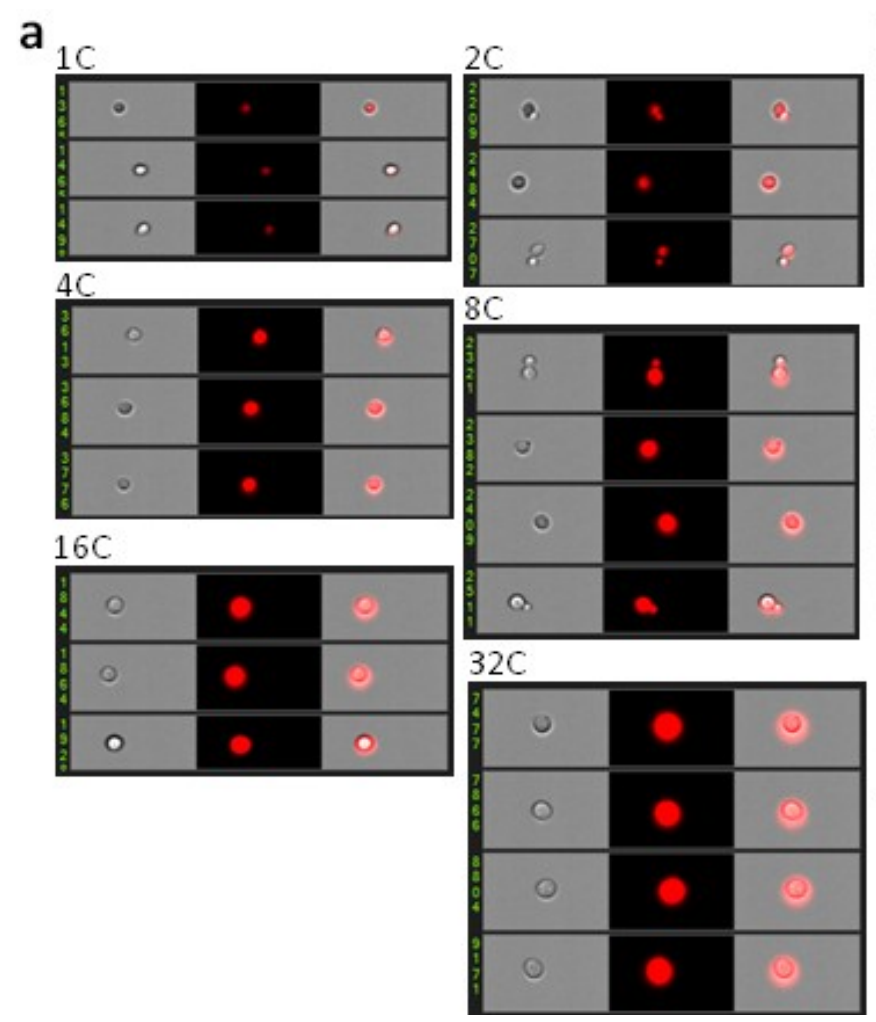

b
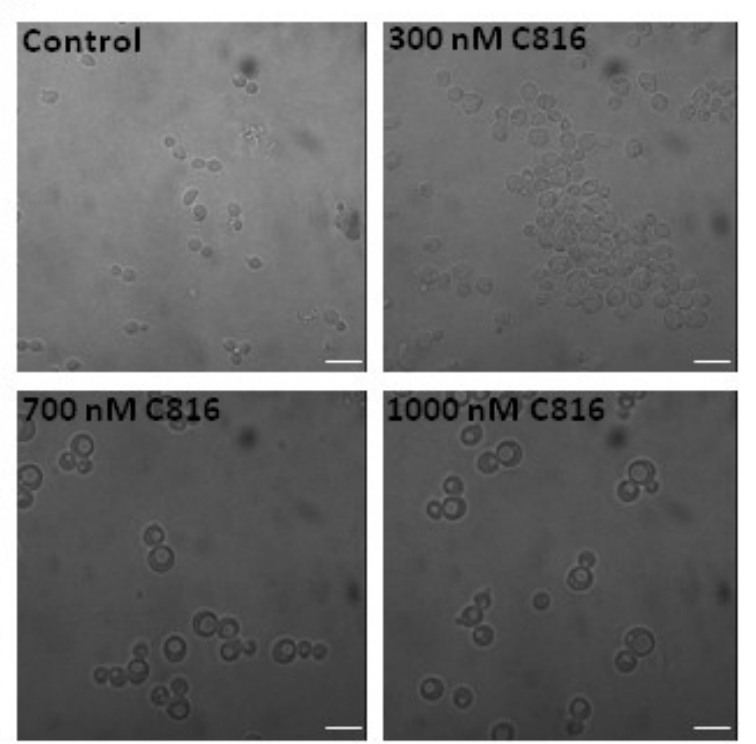


\subsection{C816 Induces Apoptosis in S. cerevisiae}

In cdc13-1 cells (S. cerevisiae mutant for $\mathrm{CDC13}$, a telomere binding protein that prevent degradation of telomeres) grown at restrictive temperature, it has been shown that increased cell size can result in cell lysis. These cells maintain high viability for several hours during which time continue to grow increasing two- to three-fold in diameter. When such cells reach a critical size, they lyse [24]. To assay if the increment in cell size induced by $\mathrm{C} 816$ could produce cell lysis, exponentially growing cultures of $S$. cerevisiae were treated with 300, 700, and $1000 \mathrm{nM} \mathrm{C816.} \mathrm{After} 10 \mathrm{~h}$ the cells were washed and then incubated in the presence of PI to determine the integrity of the plasma membrane. Treated cells did not show an increased presence of stained cells when compared to controls (Supplementary Figure S1).

After discarding the existence of necrosis and, since yeast has been shown to undergo apoptosis by numerous types of insults [25], we analyzed if C816 induced apoptosis. As shown in Figure 5b, an increase in the sub-G0/G1 cell population was observed after $24 \mathrm{~h}$ of C816 treatment (see Supplementary Figure S2 for scaled up histograms that better show this population). To better assay if C816 induced apoptosis in S. cerevisiae we performed Annexin V and PI staining experiments. Annexin V has specific affinity to phosphatidylserine (PS). Soon after apoptosis is induced, PS translocates from the inner to the outer leaflet of the plasma membrane [26]. After translocation PS can readily bind Annexin V, which coupled to FITC, was detected by flow cytometry and fluorescence microscopy. PI was also used for apoptosis determination. It binds to DNA and is impermeant to live and apoptotic cells, but stains necrotic cells. Annexin V staining showed that C816 induces apoptosis in S. cerevisiae. As determined by fluorescence microscopy, after $10 \mathrm{~h}$ there was an important increase of Annexin V positive cells without PI staining, indicative of cells in early apoptosis, while after $24 \mathrm{~h}$ we observed Annexin V staining and the appearance of PI positive cells indicative of cells in late apoptosis (secondary necrotic) (Figure 7a). When cells were analyzed for Annexin V and PI staining by flow cytometry, we observed mainly a dose dependent increase in Annexin V stained cells after $10 \mathrm{~h}$ of C816 treatment (Figure $7 \mathrm{~b}, \mathrm{c}$ ). At this time, a dose dependent reduction in cell survival was detected (Figure 7d). Supplementary Figure S3 shows representative images for healthy, Annexin V stained cells, and Annexin V-PI stained cells. As previously stated, apoptotic-like cell death has been previously described in $S$. cerevisiae after treatment with nocodazole [17]. Also, several other antineoplastic and antifungal drugs, some of which are actually used in the clinic like Amphotericin B, have already been demonstrated to induce apoptosis in yeast [27].

After apoptosis determination by fluorescence microscopy and flow cytometry, similar results were obtained showing an increase in the apoptotic population after $10 \mathrm{~h}$. After a longer incubation period, PI staining was observed indicative of secondary necrosis. In unicellular eukaryotes, the apoptosis outcome is secondary necrosis were cytoplasmic membrane permeabilization occurs and PI staining is detected. This type of necrosis was named as being secondary to distinguish this mode of cell elimination from cellular necrosis occurring without apoptosis, termed primary necrosis [28]. 
Figure 7. (a) Annexin V and PI staining of S. cerevisiae after C816 treatment for 10 and 24 h. Green: Annexin V. Red: PI. After $10 \mathrm{~h}$ only Annexin V staining is observed. PI staining is observed but after $24 \mathrm{~h}$ of treatment. Scale bar $=10 \mu \mathrm{m}$ (b) Apoptosis determination by flow cytometry in $S$. cerevisiae after C816 treatment for $10 \mathrm{~h}$. The toxin induced a dose dependent increase in Annexin V stained cells. Representative results are shown. (c) Quantification of Annexin V positive cells detected by flow cytometry. * Significant differences respect to control $p<0.01, n=3$. (d) Survival plating assay for cells treated as those analyzed by flow cytometry. After C816 treatment for $10 \mathrm{~h}$, cells were serially diluted, plated and incubated at $30{ }^{\circ} \mathrm{C}$. Colonies were counted after $48 \mathrm{~h}$. * Significant differences respect to control $p<0.01, n=3$, ** significant differences respect to control $p<0.05, n=3$.
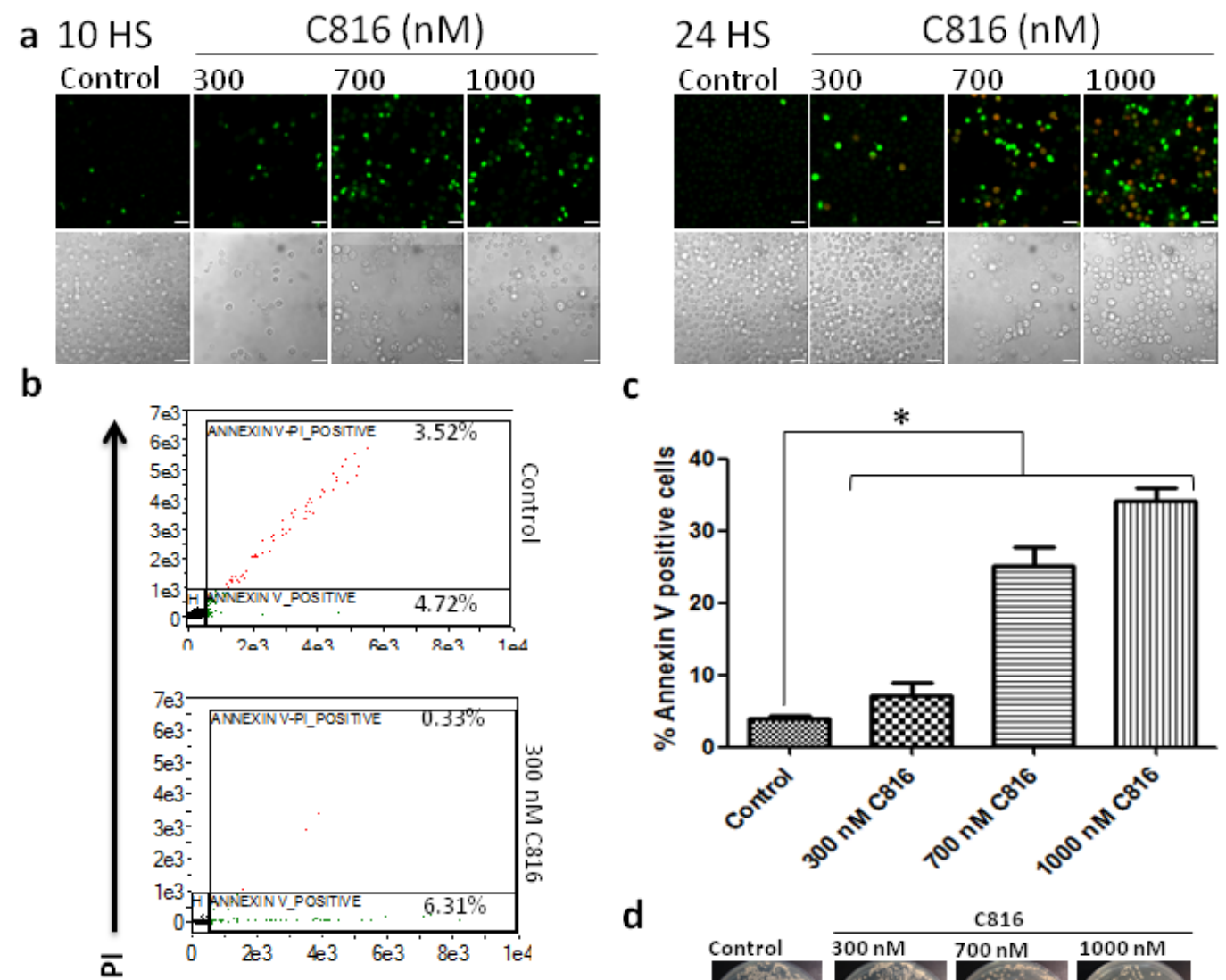

C

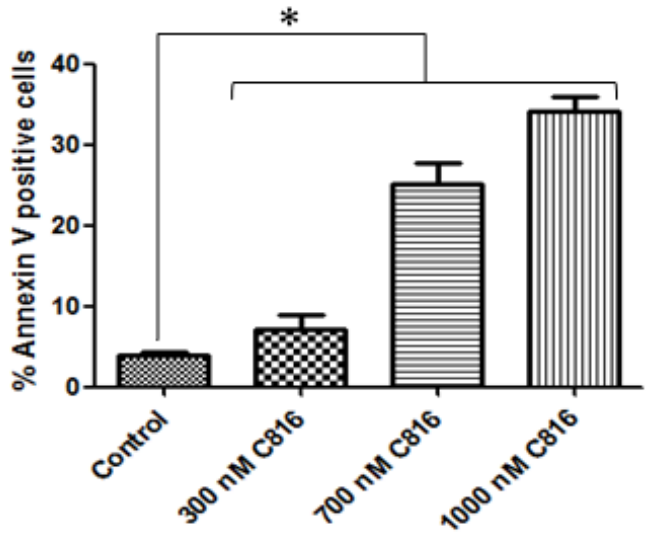

d
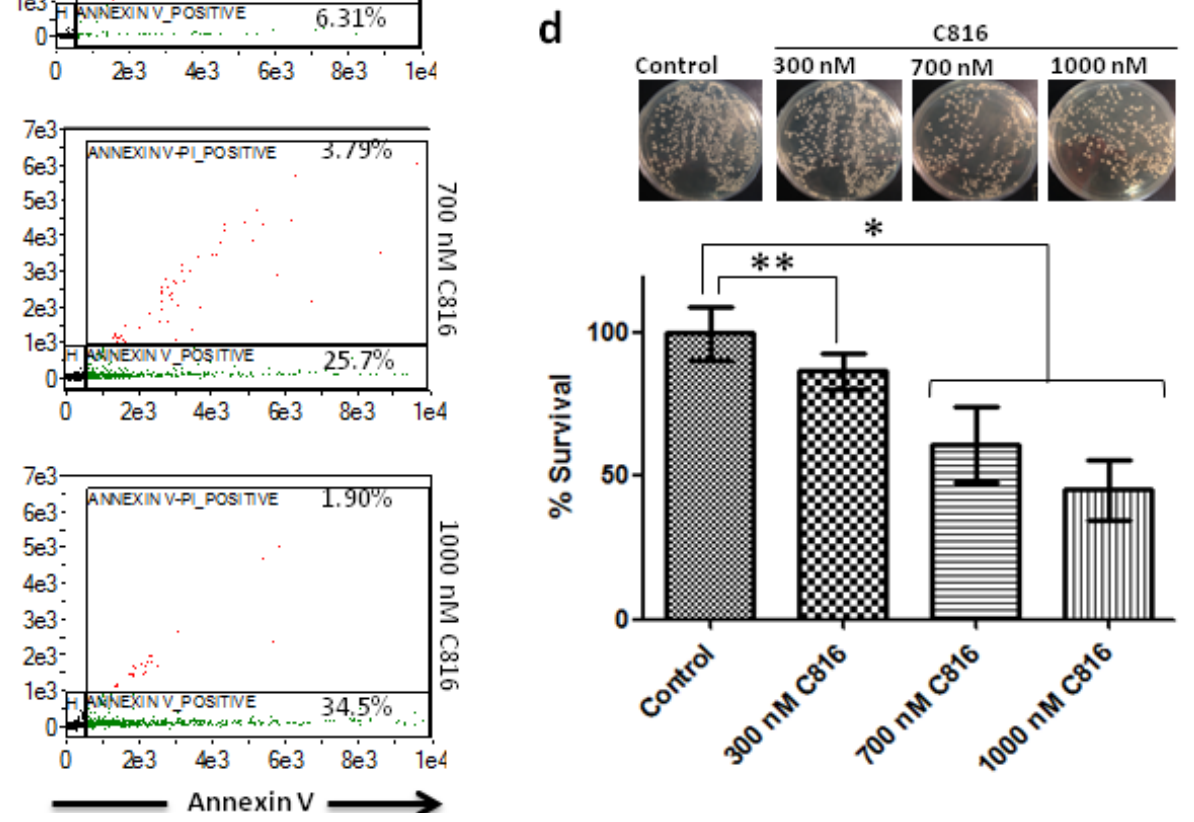


\subsection{C816 Disrupts the Mitochondrial Membrane Potential $\left(\Delta \Psi_{m}\right)$ in S. cerevisiae}

Mitochondrial permeability transition is a critical step in the cellular apoptotic pathway [29], it precedes phosphatidylserine flip-flop and coincides with caspase activation [30]. This process produces the disruption of the electrochemical gradient in the mitochondria by pore formation [31]. The basic molecular machinery of programmed cell death has been shown to be phylogenetically conserved in yeast as well as animals [25]. Several compounds, like acetic acid [32,33], $\mathrm{H}_{2} \mathrm{O}_{2}$ [34], and aspirin [35] have been shown to induce apoptosis, involving mitochondrial dysfunction, in yeast. To determine if C816 affected the mitochondrial $\Delta \Psi_{\mathrm{m}}$, we treated $S$. cerevisiae with this compound for $6 \mathrm{~h}$, the incubation period at which the highest ploidy was detected after exposure to 700 and $1000 \mathrm{nM}$ $\mathrm{C} 816$, and then analyzed the mitochondrial function using the JC-1. This dye, in the presence of high mitochondrial $\Delta \Psi_{\mathrm{m}}$, forms aggregates with orange fluorescence. In cells with damaged mitochondria JC-1 remains in the monomeric form, which shows only green fluorescence [36]. After analysis by imaging flow cytometry the results showed that C816 reduced the mitochondrial $\Delta \Psi_{\mathrm{m}}$ at the two highest concentrations tested (Figure 8a). Even though $300 \mathrm{nM} \mathrm{C816}$ appeared to decrease the mitochondrial $\Delta \Psi_{\mathrm{m}}$, the difference was not statistically significant. Examples of cells with healthy and damaged mitochondria detected after imaging flow cytometry are shown in Figure $8 \mathrm{~b}$.

Figure 8. (a) Quantification of the relative mitochondrial fluorescence (RMF) in C816 treated S. cerevisiae. * Significant differences respect to control $p<0.01, n=3$. (b) Images obtained by imaging flow cytometry of cells with functional and damaged mitochondria. SS: Side scatter, Bf: Bright field, GF: Green fluorescence, OF: Orange fluorescence, and GOBfC: Composite of the bright field, green and red fluorescence.
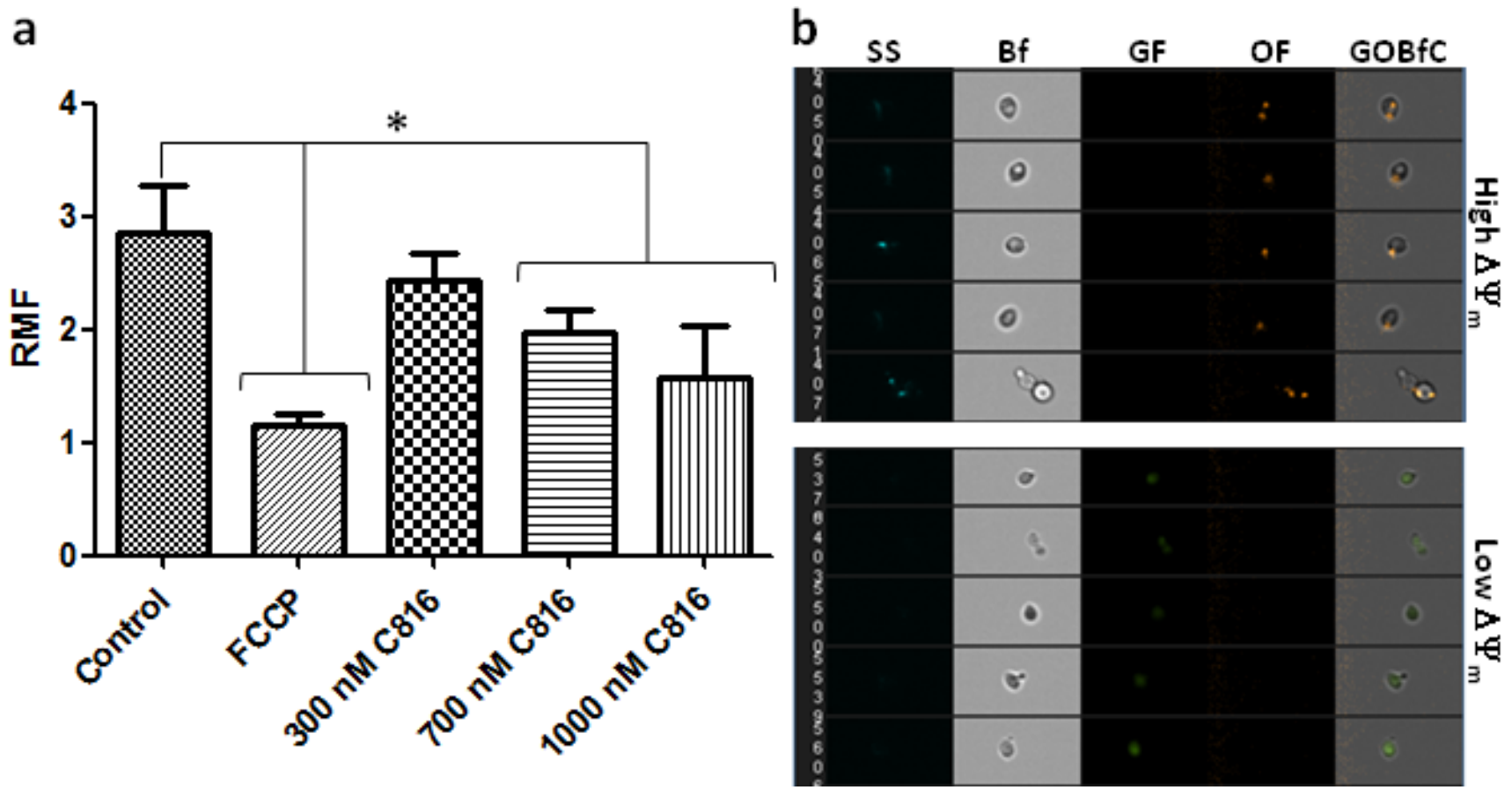

Increase in DNA content and mitochondrial depolarization with the consequent apoptosis could be related since hyperploid cells, which show an increased size, also show loss of mitochondrial membrane potential. We are currently trying to determine if this is the case. 
Saccharomyces cerevisiae, which is closely related to fungi harmful for humans [37], has been shown to be a good model system for studying the properties of antifungal compounds. Besides this, yeast has been used to study antifungals against more evolutionary distant fungi (e.g., filamentous fungi). The majority of the currently used antifungal drugs are active against $S$. cerevisiae making it a suitable model for both drug development and the elucidation of the mechanisms underlying drug's action [15]. In this work we show that C816 is a fungicidal and induces apoptosis in $S$. cerevisiae making it a potential candidate to be used as an antifungal or as a lead molecule for antifungal development.

\section{Experimental Section}

\subsection{Molecules Tested}

Crambescidin-816, -800 and -830 (Figure 1) were purified from the sponge Crambe crambe as described in [38]. The compounds were dissolved in DMSO and were 95\% pure (HPLC-MS). The final DMSO concentration for cell treatment was always lower than $0.2 \%$. Control cultures were treated with the highest DMSO concentration used in each experiment, to rule out any solvent interference in the results observed.

\subsection{Cell Strain and Culture}

The Saccharomyces cerevisiae strain NCPF3191 (ECACC) was used for all the experiments reported in this work. Cells were grown in YPD $(20 \mathrm{~g} / \mathrm{L}$ bacteriological peptone, $10 \mathrm{~g} / \mathrm{L}$ yeast extract, $20 \mathrm{~g} / \mathrm{L}$ glucose; SIGMA) at $30{ }^{\circ} \mathrm{C}$ with agitation. For culture maintenance, cells grown on YPD-agar (20 g/L bacteriological peptone, $10 \mathrm{~g} / \mathrm{L}$ yeast extract, $20 \mathrm{~g} / \mathrm{L}$ glucose, $15 \mathrm{~g} / \mathrm{L}$ agar; SIGMA) were regularly re-plated.

\subsection{Cell Growth Inhibition Determination}

Growth inhibition of $S$. cerevisiae in the presence of crambescidins was determined by a spectrophotometric bioassay to determine the $\mathrm{MIC}_{50}$ (the antimicrobial concentration the inhibits $50 \%$ of the growth) based on the change of absorbance at $600 \mathrm{~nm}$ of yeast cultures in 48 well plates. An overnight culture was diluted to a concentration of $3 \times 10^{7}$ cells per $\mathrm{mL}$ as determined with a hematocymeter. After dilution, $3 \times 10^{5}$ cells per well were seeded in 48 well plates and then treated with Crambescidin-816, -800 of -830 . The plates were incubated at $30{ }^{\circ} \mathrm{C}$ in a shaking incubator for $24 \mathrm{~h}$. Absorbance was determined prior to treatment and up to $24 \mathrm{~h}$ of incubation with shacking at $30{ }^{\circ} \mathrm{C}$, in a SYNERGY 4 spectrophometer (BIO-TEK).

\subsection{Determination of Fungicidal Effect}

The fungicidal effect of C816 was determined as previously described [39] with modifications. In brief, overnight cultures of $S$. cerevisiae were diluted to $1 \times 10^{6}$ cell $/ \mathrm{mL}$ in YPD media and incubated in 48 well plates in the presence of $\mathrm{C} 816$ or vehicle for 3 or $6 \mathrm{~h}$ at $30{ }^{\circ} \mathrm{C}$ with agitation. After incubation with the compound, cells were centrifuged and washed twice with YPD. Cells were 
resuspended in YPD media and cultured in 48 well plates up to $24 \mathrm{~h}$ at $30{ }^{\circ} \mathrm{C}$ with agitation. The optical density at $600 \mathrm{~nm}$ was determined as a measure of cell growth after the $24 \mathrm{~h}$ incubation elapsed.

To determine cell survival after C816 treatment we also performed serial dilutions of control and treated cultures and plated the cells on YPD-agar plates. Cells were incubated for $48 \mathrm{~h}$ at $30{ }^{\circ} \mathrm{C}$ and colonies were counted. Each treatment was assayed in triplicate to determine the percentage of cell survival in C816 treated cultures respect to controls.

\subsection{Cell Cycle Assay by Flow Cytometry}

An overnight culture of $S$. cerevisiae was diluted to $5 \times 10^{6}$ cell per milliliter and incubated in 48 well plates in the presence of crambescidin- 816 for 2, 4, 6, 10 and $24 \mathrm{~h}$. After each incubation, cells were washed twice with PBS and then fixed with $70 \%$ ice cold ethanol for $1 \mathrm{~h}$ at $4{ }^{\circ} \mathrm{C}$. Fixed cells were washed twice with PBS and then incubated with $10 \mu \mathrm{g} / \mathrm{mL}$ propidium iodide (PI, SIGMA) and $0.1 \mathrm{mg} / \mathrm{mL}$ RNAse (SIGMA) dissolved in PBS for $1 \mathrm{~h}$. Stained cells were sonicated for $1 \mathrm{~min}$ and 10,000 events for each sample were acquired with an AMNIS imaging flow cytometer using the AMNIS INSPIRE ${ }^{\mathrm{TM}}$ software. Data analysis was performed with the AMNIS IDEAS ${ }^{\mathrm{TM}}$ software. The experiments were performed by triplicate.

\subsection{Determination of Apoptosis by Annexin V and Propidium Iodide (PI) Staining}

To perform the assay, an overnight culture of $S$. cerevisiae was diluted to $5 \times 10^{6}$ cell per milliliter and incubated in 48 well plates in the presence of crambescidin-816 for 10 or $24 \mathrm{~h}$. After treatment, Annexin V staining was performed as previously described [40]. In brief, cells were washed with sorbitol wash buffer (1.2 M sorbitol, $0.5 \mathrm{mM} \mathrm{MgCl}_{2}, 35 \mathrm{mM}$ potassium phosphate, $\mathrm{pH}$ 6.8) and then digested with $20 \mathrm{U} / \mathrm{mL}$ of lyticase dissolved in sorbitol wash buffer for $1 \mathrm{~h}$ at $28{ }^{\circ} \mathrm{C}$. Cells were then washed with sorbitol binding buffer (10 mM HEPES, $140 \mathrm{mM} \mathrm{NaCl}, 2.5 \mathrm{mM} \mathrm{CaCl}_{2}, \mathrm{pH}$ 7.4). The cells were resuspended in $40 \mu \mathrm{L}$ of sorbitol binding buffer and two microliters of Annexin V (IMMUNOSTEP) and $1 \mu \mathrm{L}$ of $500 \mu \mathrm{g} / \mathrm{mL}$ PI were added. Cells were stained for $20 \mathrm{~min}$ at room temperature in the dark and then washed in sorbitol binding buffer. Finally the cells were resuspended in sorbitol binding buffer and observed under a fluorescence microscope, or analyzed by flow cytometry.

\subsection{Determination of Mitochondrial Transmembrane Potential $\left(\Delta \Psi_{m}\right)$}

Changes in mitochondrial $\Delta \Psi_{\mathrm{m}}$ were determined as previously described [41]. Control and C816 treated cells were washed with PBS and then resuspended in PBS with $2 \mu \mathrm{g} / \mathrm{mL} \mathrm{JC}-1$ (5,5',6,6'-tetrachloro-1,1',3,3'-tetraethylbenzimidazolylcarbocyanine iodide) and $2 \%$ glucose. Cells were stained for $30 \mathrm{~min}$ at $37{ }^{\circ} \mathrm{C}$ in the dark. After staining, cells were washed with PBS, resuspended in PBS supplemented with $2 \%$ glucose and analyzed by imaging flow cytometry. To perform compensation and as a positive control for mitochondrial uncoupling, FCCP (carbonyl cyanide 4-(trifluoromethoxy) phenylhydrazone) treated $S$. cerevisiae were used. This compound is a protonophore that uncouples oxidation from phosphorylation in mitochondria. FCCP, $10 \mu \mathrm{M}$ final concentration, was added at the same time as JC-1. Ten thousand events were acquired of each sample detecting fluorescence in the green and orange channels. The relative mitochondrial fluorescence 
(RMF) was defined as the ratio aggregated JC-1 (orange):Monomeric JC-1 (green), which reflects the changes in $\Delta \Psi_{\mathrm{m}}$ per unit of mitochondrial mass.

\subsection{Statistics}

The results were analyzed using the SIGMAPLOT ${ }^{\circledR}$ software. One way ANOVA was used for comparison of differences among groups. The Holm-Sidak multiple-range test was used for comparisons of differences between groups. A $p<0.05$ was considered significant.

\section{Conclusion}

In conclusion, we show that C816 has a fungicidal effect in yeast and is more potent than C830 and the previously postulated lead for antifungal development C800. S. cerevisiae in the presence of C816 increases its size as a consequence of higher DNA content. The most potent compound tested induced mitochondrial dysfunction and apoptosis in yeast as determined by the appearance of a sub-G0/G1 population, phosphatidylserine translocation and collapse of the mitochondrial $\Delta \Psi_{\mathrm{m}}$ in treated cells. This work indicates that C816 could be a good lead for antifungal development.

\section{Acknowledgments}

The research leading to these results has received funding from the following FEDER cofunded-grants: From Ministerio de Ciencia y Tecnología, Spain: AGL2009-13581-CO2-01, AGL2012-40485-CO2-01. From Xunta de Galicia, Spain: 10PXIB261254 PR. From the European Union's Seventh Framework Programme managed by REA-Research Executive Agency (FP7/2007-2013) under grant agreement Nos. 211326-CP (CONffIDENCE), 265896 BAMMBO, $265409 \mu \mathrm{AQUA}$, and 262649 BEADS, 315285 Ciguatools and 312184 PharmaSea. From the Atlantic Area Programme (Interreg IVB Trans-national): 2009-1/117 Pharmatlantic.

\section{Conflicts of Interest}

The authors declare no conflict of interest.

\section{References}

1. Laport, M.S.; Santos, O.C.; Muricy, G. Marine sponges: potential sources of new antimicrobial drugs. Curr. Pharm. Biotechnol. 2009, 10, 86-105.

2. Sagar, S.; Kaur, M.; Minneman, K.P. Antiviral lead compounds from marine sponges. Mar. Drugs 2010, 8, 2619-2638.

3. Abad, M.J.; Bedoya, L.M.; Bermejo, P. Marine Compounds and their Antimicrobial Activities. In Science against Microbial Pathogens: Communicating Current Research and Technological Advances; Méndez-Vilas, A., Ed.; FORMATEX: Badajoz, Spain, 2011. 
4. Mayer, A.M.; Hamann, M.T. Marine pharmacology in 2001-2002: Marine compounds with anthelmintic, antibacterial, anticoagulant, antidiabetic, antifungal, anti-inflammatory, antimalarial, antiplatelet, antiprotozoal, antituberculosis, and antiviral activities; affecting the cardiovascular, immune and nervous systems and other miscellaneous mechanisms of action. Comp. Biochem. Physiol. C Toxicol. Pharmacol. 2005, 140, 265-286.

5. Mayer, A.M.; Rodriguez, A.D.; Berlinck, R.G.; Hamann, M.T. Marine pharmacology in 2003-4: Marine compounds with anthelmintic antibacterial, anticoagulant, antifungal, anti-inflammatory, antimalarial, antiplatelet, antiprotozoal, antituberculosis, and antiviral activities; affecting the cardiovascular, immune and nervous systems, and other miscellaneous mechanisms of action. Comp. Biochem. Physiol. C Toxicol. Pharmacol. 2007, 145, 553-581.

6. Mayer, A.M.; Rodriguez, A.D.; Berlinck, R.G.; Hamann, M.T. Marine pharmacology in 2005-6: Marine compounds with anthelmintic, antibacterial, anticoagulant, antifungal, anti-inflammatory, antimalarial, antiprotozoal, antituberculosis, and antiviral activities; affecting the cardiovascular, immune and nervous systems, and other miscellaneous mechanisms of action. Biochim. Biophys. Acta 2009, 1790, 283-308.

7. Buscema, M.; Van de Vyver, G. Cytotoxic rejection of xenografts between marine sponges. J. Exp. Zool. 1985, 235, 297-308.

8. Burkholder, P.R.; Ruetzler, K. Antimicrobial activity of some marine sponges. Nature 1969, 222, 983-984.

9. Uriz, M.J.; Beccero, M.A.; Tur, J.M.; Turon, X. Location of toxicity within the Mediterranean sponge Crambe crambe. Mar. Biol. 1996, 124, 583-590.

10. Aron, Z.D.; Pietraszkiewicz, H.; Overman, L.E.; Valeriote, F.; Cuevas, C. Synthesis and anticancer activity of side chain analogs of the crambescidin alkaloids. Bioorg. Med. Chem. Lett. 2004, 14, 3445-3449.

11. Aoki, S.; Kong, D.; Matsui, K.; Kobayashi, M. Erythroid differentiation in K562 chronic myelogenous cells induced by crambescidin 800 , a pentacyclic guanidine alkaloid. Anticancer Res. 2004, 24, 2325-2330.

12. Gassner, N.C.; Tamble, C.M.; Bock, J.E.; Cotton, N.; White, K.N.; Tenney, K.; St Onge, R.P.; Proctor, M.J.; Giaever, G.; Nislow, C.; et al. Accelerating the discovery of biologically active small molecules using a high-throughput yeast halo assay. J. Nat. Prod. 2007, 70, 383-390.

13. Berlinck, R.G.; Braekman, J.C.; Daloze, D.; Bruno, I.; Riccio, R.; Ferri, S.; Spampinato, S.; Speroni, E. Polycyclic guanidine alkaloids from the marine sponge Crambe crambe and $\mathrm{Ca}++$ channel blocker activity of crambescidin 816. J. Nat. Prod. 1993, 56, 1007-1015.

14. Martin, V.; Vale, C.; Bondu, S.; Thomas, O.P.; Vieytes, M.R.; Botana, L.M. Differential Effects of Crambescins and Crambescidin 816 in Voltage-Gated Sodium, Potassium and Calcium Channels in Neurons. Chem. Res. Toxicol. 2013, in press.

15. Hughes, T.R. Yeast and drug discovery. Funct. Integr. Genomics 2002, 2, 199-211.

16. Pichler, S.; Piatti, S.; Nasmyth, K. Is the yeast anaphase promoting complex needed to prevent re-replication during G2 and M phases? EMBO J. 1997, 16, 5988-5997.

17. Endo, K.; Mizuguchi, M.; Harata, A.; Itoh, G.; Tanaka, K. Nocodazole induces mitotic cell death with apoptotic-like features in Saccharomyces cerevisiae. FEBS Lett. 2010, 584, 2387-2392. 
18. Hartwell, L.H. Genetic control of the cell division cycle in yeast. IV. Genes controlling bud emergence and cytokinesis. Exp. Cell Res. 1971, 69, 265-276.

19. Cid, V.J.; Adamikova, L.; Cenamor, R.; Molina, M.; Sanchez, M.; Nombela, C. Cell integrity and morphogenesis in a budding yeast septin mutant. Microbiology 1998, 144, 3463-3474.

20. Longtine, M.S.; DeMarini, D.J.; Valencik, M.L.; Al-Awar, O.S.; Fares, H.; De Virgilio, C.; Pringle, J.R. The septins: Roles in cytokinesis and other processes. Curr. Opin. Cell Biol. 1996, 8, 106-119.

21. Howell, A.S.; Lew, D.J. Morphogenesis and the cell cycle. Genetics 2012, 190, 51-77.

22. Lew, D.J.; Reed, S.I. Cell cycle control of morphogenesis in budding yeast. Curr. Opin. Genet. Dev. 1995, 5, 17-23.

23. Rubiolo, J.A.; López-Alonso, H.; Roel, M.; Thomas, O.P.; Ternon, E.; Vieytes, M.R.; Vega, F.V.; Botana, L.M. Mechanism of cytotoxic action of crambescidin-816 on liver-derived tumour cell lines. Brit. J. Pharm. 2013, submitted for publication.

24. Wysocki, R.; Kron, S.J. Yeast cell death during DNA damage arrest is independent of caspase or reactive oxygen species. J. Cell Biol. 2004, 166, 311-316.

25. Madeo, F.; Herker, E.; Wissing, S.; Jungwirth, H.; Eisenberg, T.; Frohlich, K.U. Apoptosis in yeast. Curr. Opin. Microbiol. 2004, 7, 655-660.

26. van Engeland, M.; Nieland, L.J.; Ramaekers, F.C.; Schutte, B.; Reutelingsperger, C.P. Annexin V-affinity assay: A review on an apoptosis detection system based on phosphatidylserine exposure. Cytometry 1998, 31, 1-9.

27. Almeida, B.; Silva, A.; Mesquita, A.; Sampaio-Marques, B.; Rodrigues, F.; Ludovico, P. Drug-induced apoptosis in yeast. Biochim. Biophys. Acta 2008, 1783, 1436-1448.

28. Silva, M.T. Secondary necrosis: The natural outcome of the complete apoptotic program. FEBS Lett. 2010, 584, 4491-4499.

29. Desagher, S.; Osen-Sand, A.; Nichols, A.; Eskes, R.; Montessuit, S.; Lauper, S.; Maundrell, K.; Antonsson, B.; Martinou, J.C. Bid-induced conformational change of Bax is responsible for mitochondrial cytochrome c release during apoptosis. J. Cell Biol. 1999, 144, 891-901.

30. Korper, S.; Nolte, F.; Rojewski, M.T.; Thiel, E.; Schrezenmeier, H. The K+ channel openers diazoxide and NS1619 induce depolarization of mitochondria and have differential effects on cell Ca2+ in CD34+ cell line KG-1a. Exp. Hematol. 2003, 31, 815-823.

31. Narita, M.; Shimizu, S.; Ito, T.; Chittenden, T.; Lutz, R.J.; Matsuda, H.; Tsujimoto, Y. Bax interacts with the permeability transition pore to induce permeability transition and cytochrome $\mathrm{c}$ release in isolated mitochondria. Proc. Natl. Acad. Sci. USA 1998, 95, 14681-14686.

32. Giannattasio, S.; Atlante, A.; Antonacci, L.; Guaragnella, N.; Lattanzio, P.; Passarella, S.; Marra, E. Cytochrome $\mathrm{c}$ is released from coupled mitochondria of yeast en route to acetic acid-induced programmed cell death and can work as an electron donor and a ROS scavenger. FEBS Lett. 2008, 582, 1519-1525.

33. Ludovico, P.; Rodrigues, F.; Almeida, A.; Silva, M.T.; Barrientos, A.; Corte-Real, M. Cytochrome c release and mitochondria involvement in programmed cell death induced by acetic acid in Saccharomyces cerevisiae. Mol. Biol. Cell 2002, 13, 2598-2606. 
34. Pereira, C.; Camougrand, N.; Manon, S.; Sousa, M.J.; Corte-Real, M. ADP/ATP carrier is required for mitochondrial outer membrane permeabilization and cytochrome c release in yeast apoptosis. Mol. Microbiol. 2007, 66, 571-582.

35. Sapienza, K.; Bannister, W.; Balzan, R. Mitochondrial involvement in aspirin-induced apoptosis in yeast. Microbiology 2008, 154, 2740-2747.

36. Smiley, S.T.; Reers, M.; Mottola-Hartshorn, C.; Lin, M.; Chen, A.; Smith, T.W.; Steele, G.D., Jr.; Chen, L.B. Intracellular heterogeneity in mitochondrial membrane potentials revealed by a J-aggregate-forming lipophilic cation JC-1. Proc. Natl. Acad. Sci. USA 1991, 88, 3671-3675.

37. Barns, S.M.; Lane, D.J.; Sogin, M.L.; Bibeau, C.; Weisburg, W.G. Evolutionary relationships among pathogenic Candida species and relatives. J. Bacteriol. 1991, 173, 2250-2255.

38. Bondu, S.; Genta-Jouve, G.; Leirós, M.; Vale, C.; Guigonis, J.-M.; Botana, L.M.; Thomas, O.P. Additional bioactive guanidine alkaloids from the Mediterranean sponge Crambe crambe. RSC Adv. 2012, 2, 2828-2835.

39. Tani, N.; Rahnasto-Rilla, M.; Wittekindt, C.; Salminen, K.A.; Ritvanen, A.; Ollakka, R.; Koskiranta, J.; Raunio, H.; Juvonen, R.O. Antifungal activities of novel non-azole molecules against S. cerevisiae and C. albicans. Eur. J. Med. Chem. 2012, 47, 270-277.

40. Madeo, F.; Frohlich, E.; Frohlich, K.U. A yeast mutant showing diagnostic markers of early and late apoptosis. J. Cell Biol. 1997, 139, 729-734.

41. Pina-Vaz, C.; Sansonetty, F.; Rodrigues, A.G.; Costa-Oliveira, S.; Tavares, C.; Martinez-de-Oliveira, J. Cytometric approach for a rapid evaluation of susceptibility of Candida strains to antifungals. Clin. Microbiol. Infect. 2001, 7, 609-618.

(C) 2013 by the authors; licensee MDPI, Basel, Switzerland. This article is an open access article distributed under the terms and conditions of the Creative Commons Attribution license (http://creativecommons.org/licenses/by/3.0/). 